\title{
Stockage en nappe profonde à haute température Pilote de Plaisir
}

\author{
Storage in a deep aquifer at high temperature \\ Pilot plant in Plaisir
}

\author{
J. Despois \\ Commissariat à l'Energie Atomique \\ IRDI-DEDR-DEMT - Centre d'Etudes Nucléaires de Saclay
}

\begin{abstract}
Le stockage souterrain de chaleur constitue avec la source thermique et l'utilisation un ensemble indissociable qui doit étre optimisé. Ceci impose des contraintes sur la taille et la température du stockage et, par là, sur le choix des nappes. Il existe, pour chaque site, une température optimale d'injection, généralement très supérieure à $100^{\circ} \mathrm{C}$. On peut alors espérer une récupération d'environ $60 \%$ de la chaleur stockée, utilisable sur des réseaux classiques de distribution. Les problèmes technologiques qui en résultent paraissent résolus.

La construction d'un stockage pilote couplé à une usine d'incinération d'ordures traitant 120000 tonnes/an et à un réseau desservant plus de 5000 logements commence à Plaisir, près de Versailles.
\end{abstract}

Underground heat storage forms an indissociable system with the heat source and use and must be optimized. This imposes constraints on the size and the storage temperature and thereby on the selection of the water tables. For each site, there is an optimum injection temperature, generally substantially above $100{ }^{\circ} \mathrm{C}$. It can then be expected to recover approximately 60 percent of the heat stored for use on conventional distribution systems. The related engineering problems appear to be solved.

The construction of a pilot storage facility coupled with a garbage incineration plant processing 120000 metric tons/year and a system serving more than 5000 dwellings has been started in Plaisir, near Versailles.

\section{La démarche adoptée}

Depuis 1973, les études portant sur le stockage souterrain de chaleur se sont développées un peu partout dans le monde.

L'objectif initial était le stockage de modestes quantités de chaleur à bas niveau de température, pour le chauffage de petits ensembles immobiliers ou de serres.

Ces travaux ont apporté de nombreux renseignements scientifiques de grand intérêt. Mais, nulle part, les résultats techniques et économiques ne se sont révélés suffisants pour permettre une généralisation du procédé, aussi longtemps que l'on s'en est tenu aux objectifs initiaux.

Ceci a conduit certaines équipes à rechercher une amélioration des performances dans deux directions opposées :

1) En abaissant le niveau de température, le stockage peut se faire à très faible profondeur et se transforme en un système de régénération thermique des nappes exploitées comme sources de pompes à chaleur. Il ne faut pas, en effet, chercher à relever systématiquement la température de récupération: celle-ci, demeurant faible de toutes façons, on ne peut attendre de ce relèvement qu'une légère amélioration du coefficient de performance des pompes à chaleur, peu susceptible de justifier les surinvestissements et les surcoûts de fonctionnement que nécessiterait le choix de températures plus élevées. Dans un tel système, la notion de rendement devient secondaire et mal définie. Pour une opération bien conçue, le succès économique n'est pas inconcevable. L'opération d'Aulnay-sous-Bois montre, au moins, qu'il ne s'agit pas d'une utopie.

2) En élevant le niveau de température de stockage et en accroissant la taille du système, on peut espérer une amélioration des performances. Cette voie a été suivie aux 
Etats-Unis et des projets existent au Danemark et en France. Les résultats américains montrent que, au voisinage de $100^{\circ} \mathrm{C}$, les contraintes chimiques demeurent acceptables et le bilan thermique est meilleur qu'aux températures plus basses. L'inconvénient de cette solution est évidemment qu'elle exclut le couplage à des sources de " chaleur douce" .

En outre, du fait de la démarche adoptée, les systèmes envisagés ne sont que des adaptations de dispositifs initialement prévus pour des températures plus basses. La chaleur provenant de sources à température élevée, comme les usines d'incinération d'ordures ménagères, doit être dégradée avant son stockage, en raison des limitations technologiques. Ceci pèse lourdement sur les performances de dispositifs qui ne constituent pas, avec la source et le réseau utilisateur, des ensembles globalement optimisés. C'est probablement pourquoi le procédé trouve difficilement des débouchés commerciaux.

Le Commissariat à l'énergie atomique et la société nationale ELF-Aquitaine qui, dans ce domaine, collaborent depuis une dizaine d'années, ont eu une approche du problème à l'opposé des démarches précédentes. En admettant d'emblée le caractère complexe du système global source-stockage-réseau, il a été recherché quel était le couplage optimal, pour différents types d'utilisation. On peut ainsi déterminer les paramètres idéaux pour chacun des éléments : taille et températures de la source, du stockage et du réseau.

Il est clair que les premières réalisations doivent se rapprocher le plus possible de ces conditions théoriques, des accommodements pouvant et devant être trouvés ultérieurement, quand les problèmes seront mieux maitrisés. Les paramètres concernant la source et le réseau permettent de déterminer le marché possible pour le stockage, marché qui n'est pas nécessairement celui que l'on aurait imaginé dans l'abstrait. A partir de là, les paramètres du stockage sont déterminés et apparaissent comme des objectifs à atteindre et non comme le résultat d'observations. Il a alors été possible de recenser les difficulter et de chercher des solutions aux problèmes ainsi posés. Il aurait été souhaitable de conforter cette analyse en engageant une expérience scientifique de grandeur significative, capable d'assurer la qualification du procédé et la possibilité d'une réalisation opérationnelle ultérieure.

Malheureusement, des considérations dimensionnelles permettent de démontrer qu'une telle expérience devrait avoir une grande taille, donc un coût voisin de celui d'une opération réelle. Pour les recherches sur la gestion de l'énergie, contrairement à ce qui se passe dans d'autres domaines, il est très difficile de trouver des financements importants pour des expériences purement scientifiques.

Bien que les sites favorables à de telles expériences ne soient pas les mêmes que les sites convenant pour une exploitation commerciale, on a dû envisager une véritable opération de démonstration assurant l'utilisation effective des intallations en cas de succès des essais. On a toutefois veillé à ce que cette opération, qui va se dérouler près de PLAISIR (Yvelines), sauvegarde l'essentiel, à savoir la recherche systématique de l'optimum technico-économique du système global source-stokage-réseau.

\section{Taille du stockage}

Il existe un besoin de stockage quand, dans le temps, la différence entre prix de vente et coût marginal de production de la chaleur varie plus que ne coûte l'opération de stockage et récupération. Il n'est pas nécessaire que la production soit fatale : les centrales nucléaires ne produisent pas d'énergie fatale, mais peuvent créer un besoin de stockage. Mais les productions fatales (usines d'incinération d'ordures) sont évidemment des cas intéressants. Encore faut-il qu'à cette production corresponde une consommation adaptée. La figure 1 montre l'évolution de la puissance appelée d'un réseau, en fonction du temps (climat de la région parisienne). Si on couple ce réseau à une usine d'incinération, on conçoit que pour une production de l'usine très grande (courbe $\mathrm{A}$ ), on pourra stocker de la chaleur, mais il n'y aura pratiquement pas de besoins de récupération. Si l'usine produit très peu (courbe $\mathrm{B}$ ), on sera dans la situation inverse, tout aussi défavorable.

Dans la région parisienne, pour un stockage permettant de récupérer 60 à $70 \%$ de la chaleur stockée, l'optimum se situe sur la courbe $\mathrm{C}$. On constate que le stockage fournit alors $30 \%$ des besoins. Ce maximum ne peut pas être dépassé. Si l'on est très au-dessous de cette valeur, il y a un surplus ou un manque de chaleur et la rentabilité du système se dégrade. En cas d'excès de chaleur, l'extension du réseau constitue un palliatif possible, mais la rentabilité marginale de ces extensions ne peut jamais être très bonne et l'on trouve vite les limites de cet expédient. La quantité de chaleur à stocker est donc imposée par les conditions de surface. Il faut alors trouver une nappe convenable; trop étendu (trop plat), le stockage fonctionnera comme un mauvais échangeur à plaques; trop peu étendu, il sera instable. L'optimum se situe pour un rayon maximal du stock utile compris entre 5 et 10 fois son épaisseur.

Il en résulte que, pour une température déterminée, les conditions de surface imposent des contraintes à l'épaisseur des nappes exploitables. En général, en France, celles-ci sont plutôt peu épaisses. Pour tendre à ramener le rayon à des valeurs acceptables, on aura intérêt à augmenter la température. Cet accroissement permettra de réduire les débits d'eau, donc de se contenter de nappes moins perméables et d'atteindre des profondeurs plus grandes avec des puits de diamètre limité : l'accroissement de la température permet donc d'augmenter les chances de trouver une nappe ayant les caractéristiques géométriques et physiques voulues. 


\section{Rendement}

On ne peut pas choisir une température sans évoquer la notion de rendement qui recouvre des réalités bien différentes :

\section{Rendement théorique global}

C'est à chaque instant ou en fin de cycle, le rapport de la somme de la chaleur récupérée et de celle restant en stock à la chaleur initialement stockée. Le rendement théorique global n'a pratiquement pas de signification pour les stockages souterrains, car, s'appuyant sur des calculs enthalpiques, il néglige la dégradation de l'énergie. Pour un stockage assez profond, on peut alors considérer un volume de terrain assez grand pour qu'il soit adiabatique, aux extractions ou injections volontaires près. Le rendement théorique global d'un stockage souterrain profond est donc constamment égal à l'unité.

\section{Rendement théorique tronqué}

C'est à chaque instant, ou en fin de cycle, le rapport de la somme de la chaleur récupérée et de la chaleur restant en stock, au-dessus d'une température de référence, à la chaleur initialement stockée. En principe, la température de référence est la température de retour du réseau utilisateur (éventuellement majorée pour tenir compte du pincement des échangeurs). Pour un réservoir de surface ayant une isolation donnée, après une durée donnée, ce rendement est une fonction souvent croissante de la température. Si la taille du réservoir est ajustée pour une quantité de chaleur donnée, à épaisseur donnée d'isolant, c'est une fonction de la température de stockage qui peut demeurer croissante. Si l'on raisonne à volume donné d'isolant cette fonction est même fortement croissante. On peut vérifier aisément ces propriétés, en opposition avec un "bon sens" un peu trivial. Ce rendement permet de suivre l'évolution du comportement d'un stock, mais il donne une vision optimiste de la réalité : ce n'est pas parce que, à un instant donné, il y a en stock de la chaleur potentiellement récupérable qu'elle le sera encore au moment où l'on en aura affectivement besoin.

\section{Rendement pratique}

C'est le rapport de la chaleur récupérée à la chaleur stockée. Il peut être calculé sur un cycle, ou cumulé depuis les origines du système. Le rendement pratique d'un cycle est nul pendant la phase de stockage et croît pendant la phase de récupération (alors que le rendement théorique tronqué décroît du début à la fin du cycle). Ce n'est qu'à la fin de chaque cycle que le rendement pratique a une valeur significative; en cours de cycle, sa valeur dépend principalement des conditions d'exploitation. Le rendement pratique est le seul rendement intéressant du point de vue économique.

On ne peut approcher le rendement pratique ni par l'intuition, ni par des calculs analytiques, ni même par des simulations ou expériences purement théoriques.

Avant un essai réel, seules des simulations prenant effectivement en compte les conditions d'exploitation permettent d'en avoir une idée raisonnée, sinon exacte.

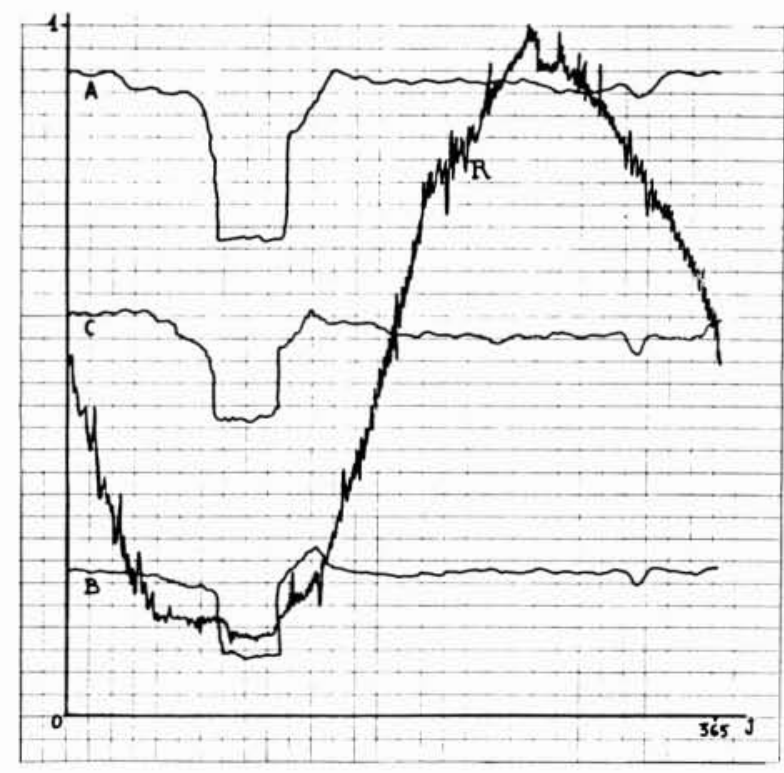

Figure 1. - Courbes chronologiques des puissances appelées par un réseau $(R)$ et des puissances fournies par une usine d'incinération (A, B, C : voir texte). Unité de puissance: puissance maximale appelée par le réseau. 


\section{Simulations d'un stockage}

Il est relativement facile de simuler le fonctionnement d'un stockage souterrain par un modèle à éléments finis, bien que l'hypothèse du milieu continu équivalent sur laquelle repose ces modèles soit, en l'occurrence, discutable. Mais, pour être représentatifs, ils doivent prendre en compte de nombreux phénomènes complexes. Même avec de puissants ordinateurs modernes comme le CRAY ils nécessitent des temps de calculs très longs et se prêtent mal aux passages multiples des études de sensibilité. Or celles-ci sont d'autant plus nécessaires que les paramètres du sous-sol sont mal connus, que l'on n'est pas à l'abri d'hétérogénéités et que les conditions précises d'exploitation présentent une large part d'aléas, notamment d'origine climatique.

Pour éviter ces inconvénients, on a choisi de faire une simulation rustique, semi-analytique, où les phénomènes non pris en compte peuvent être appréhendés globalement par des termes correctifs agissant à la manière de nombres de Nüsselt. Les temps de calcul sont alors très réduits, ce qui présente un double avantage :

- chaque simulation peut être poursuivie sur plusieurs cycles (en pratique : quatre cycles d'un an). Ceci est important dans la mesure où le premier cycle est peu significatif;

- on peut multiplier les simulations, d'abord pour vérifier l'influence d'une variation des termes correctifs, mais aussi pour tenir compte des marges d'erreurs importantes sur les paramètres de terrain, ainsi que des incertitudes sur les paramètres d'exploitation.

On calcule ainsi les rendements pratiques, les températures de récupération et différents résultats d'exploitation, comme l'énergie dépensée par les pompes.

\section{Résultats}

Les résulats peuvent, à première vue, surprendre, mais confirment la validité de la démarche retenue. Les paramètres de terrain agissent pratiquement en "tout ou rien ". - Si l'on s'écarte trop des conditions optimales, l'échec est certain et aucune intervention n'est de nature à y remédier, notamment en ce qui concerne la température de stockage.

Les paramètres d'exploitation sont, par contre, déterminants, du point de vue économique. Les rendements sont très sensibles à la température de retour du réseau utilisateur, qui doit être aussi basse que possible. Ils dépendent aussi très étroitement de la température de stockage qui doit être aussi élevée que possible.

Pour des stockages à coupler avec une usine d'incinération importante, on peut s'attendre, après 4 ans, à des rendements pratiques de 60 à $70 \%$ pour une température de stockage de $180^{\circ} \mathrm{C}$ et une température de retour du réseau de $70^{\circ} \mathrm{C}$. On assiste à un véritable effondrement de ces rendements si la température de retour du réseau dépasse $85^{\circ} \mathrm{C}$ ou si la température de stockage est de $110^{\circ} \mathrm{C}$.

\section{Choix de la température}

La température de stockage est limitée par :

- la température de la source;

- les réactions chimiques du terrain;

- la profondeur de la couche et ses caractéristiques hydrauliques (de manière à éviter l'ébullition, même au moment de l'extraction maximale qui déprime la zone chaude de la nappe);

- les contraintes technologiques.

Les études et les simulations chimiques ont montré que des perturbations sérieuses pouvaient apparaître au-dessus de $200^{\circ} \mathrm{C}$, température au-delà de laquelle la pression devient vite importante. On peut, pratiquement, se limiter à $180^{\circ} \mathrm{C}$. Mais tous les terrains ne supportent pas cette température.

Les simulations montrent seulement que, sur un site donné, le stockage doit être effectué à la température la plus élevée possible : parfois celle-ci ne dépassera pas $100^{\circ} \mathrm{C}$, mais si on le peut, il faut viser $180^{\circ} \mathrm{C}$, car on a plus de chances d'obtenir de bons résultats techniques et économiques. Encore faut-il maîtriser la technologie de ces stockages : c'est la démarche qui a été adoptée.

Ceci n'implique pas que tout l'arsenal technologique soit utilisé dans tous les cas: des dispositifs nécessaires à $180^{\circ} \mathrm{C}$ peuvent être éliminés à $120^{\circ} \mathrm{C}$; mais cette élimination ne doit pas être un prétexte à l'abaissement des températures, car elle ne compensera jamais les surcoûts résultant d'un fonctionnement à $120^{\circ} \mathrm{C}$ sur un site qui en aurait supporté $180^{\circ} \mathrm{C}$. Il n'existe donc pas de stockage à moyenne température qui s'opposeraient aux stockages à haute température, mais seulement des stockages adaptés au site, c'est-à-dire utilisant la température maximale compatible avec ce site et impliquant la nécessité de maîtriser la technologie, de manière à ce qu'elle n'introduise pas de contraintes supplémentaires dans les éléments du choix.

\section{Conséquences technologiques des températures élevées}

1) La pression doit être maîtrisée en tout point, ce qui a nécessité la mise au point d'une vanne de fond spéciale, installée dans le puits chaud de stockage et récupération.

2) La pompe immergée de récupération doit pouvoir supporter la température maximale.

3) Les dissolutions et précipitations posent des problèmes différents de ceux recontrés aux températures plus basses, sans être plus critiques (même si, pour la première opération, on a, par prudence, envisagé des possibilités de traitement d'eau très largement calculées). 


\section{L'opération de Plaisir}

\section{Objectif}

L'opération de Plaisir est un test en vraie grandeur d'un stockage optimisé en température. On ne peut pas en garantir le succès, mais on peut être assuré, en cas d'échec, qu'aucun autre stockage n'aurait donné satisfaction. Après préchauffage à température moyenne, la température sera, si possible, portée au plus près de $180^{\circ} \mathrm{C}$ en fond de puits, le site ayant été choisi pour permettre de tester l'éventail complet des températures concevables.

\section{Particularité de construction}

L'exploitation ne se fera pas par un doublet, mais par un puits chaud central, entouré de trois puits tièdes périphériques (figure 2). Ce dispositif n'est pas un impératif de la haute température, mais celle-ci permet de l'envisager plus facilement. Il y a, de cette manière, possibilité de contrôler et de corriger la position du stock, de récupérer une partie des pertes, de limiter le risque d'invasion de la base du puits chaud par de l'eau froide.

Au stockage de la chaleur, l'eau est pompée dans les puits périphériques, traitée et filtrée en surface, pressurisée et portée à $180^{\circ} \mathrm{C}$ avant d'être réinjectée dans le puits central.

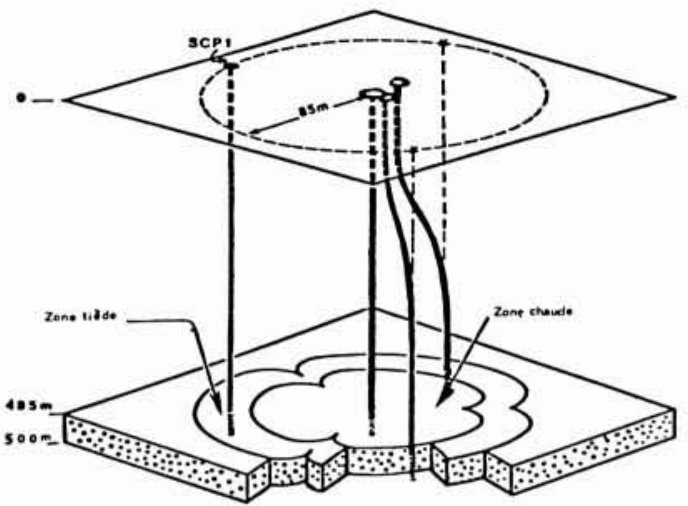

Figure 2. - Schéma du stockage de Thiverval-Grignon, couplé au réseau de PLAISIR.

SCP1 : sigle du forage de reconnaissance, déjà creusé et réutilisable comme puits périphérique.

Figure 3. - Forage de reconnaissance. Vue du chantier.

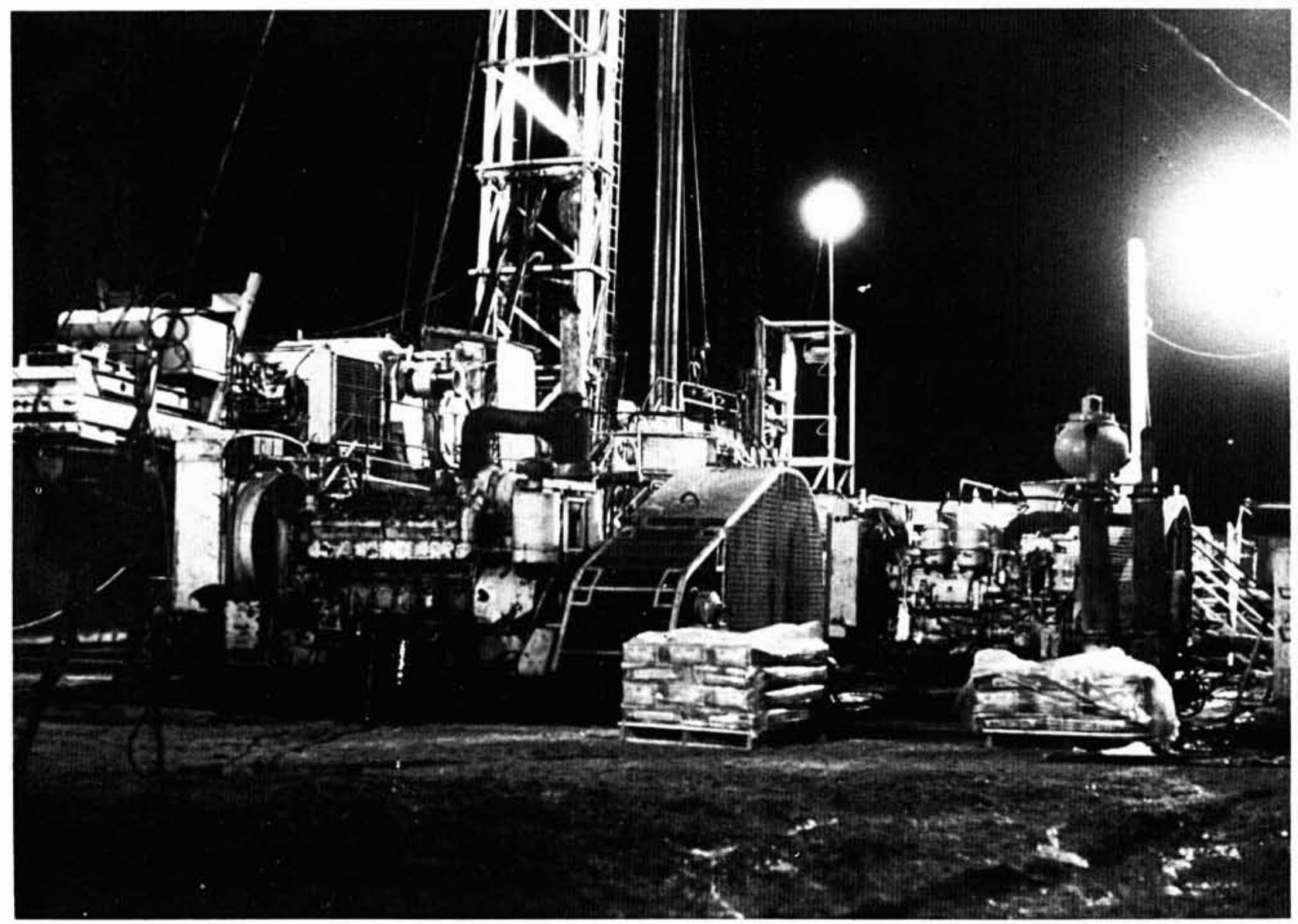


A la récupération de la chaleur, l'eau est pompée dans le puits central, passe dans un échangeur réchauffant les retours du réseau de distribution, est traitée et filtrée avant réinjection dans les puits périphériques, à une température voisine de $70^{\circ} \mathrm{C}$.

\section{Description}

L'usine d'incinération de Plaisir, d'une capacité de 140000 tonnes par an et ne fonctionnant encore qu'à $75 \%$ de cette capacité, dissipe actuellement la chaleur produite. Elle va être équipée d'un système de récupération par les soins du Syndicat Intercommunal propriétaire.

ELF-Energie et la Compagnie générale de chauffe, réunies au sein du GIE RESOP construisent un réseau de distribution desservant entre 5000 et 6000 logements, ayant une puissance maximale de $50 \mathrm{MW}$ et une consommation annuelle de $90 \mathrm{GWh}$.

La société nationale ELF-Aquitaine et le Commissariat à l'énergie atomique assurent la construction et les essais du stockage souterrain, situé à 500 mètres de profondeur dans un horizon sableux du Wealdien, épais de 15 mètres environ.
En l'absence de stockage, le réseau aurait été moins étendu et l'économie annuelle serait limitée à 6900 Tep. Si le stockage fonctionne comme prévu, l'économie dépasse $10000 \mathrm{Tep}$, avec un taux de couverture des besoins des utilisateurs dépassant $90 \%$. L'opération bénéficie d'aides de la Communauté économique européenne et de l'Agence française pour la maitrise de l'énergie.

\section{Situation actuelle et prévisions}

Un forage de reconnaissance réutilisable comme puits périphérique tiède a été foré et équipé (figure 3).

Les travaux de génie civil sont terminés.

Les forages et la construction des installations de surface du stockage (qui ne sont pas sur le " chemin critique ") vont entrer dans une phase active au printemps de 1985 , mais la première mise en chaleur ne pourra pas intervenir avant 1986, en raison des délais d'équipement de l'usine d'incinération. Les premiers résultats significatifs ne pourront guère être obtenus avant 1987, ou, plus probablement, 1988. Un bilan complet peut être attendu pour 1990.

\section{Discussion}

Pourquoi trois puits ? L'idéal serait une infinité de puits, un cercle de puits. C'est uniquement pour des raisons économiques qu'on s'est limité à trois puits. Il s'agit de faire, en somme, des feuilles de trèfle (question de $M$. THIRRIOT).

$\mathrm{Ce}$ dispositif permet de limier les dérives du stockage en fonctionnement normal ou sous les effets de perturbations antérieures. Par exemple celles dues au stockage de gaz de Beynes situé à 5 ou $6 \mathrm{~km}$ dans le même horizon aquifère. Le stockage d'eau chaude n'a pas d'effet sur le stockage de gaz (question de M. SAUTr) mais l'inverse n'est pas vrai. En jouant sur les débits des différents puits périphériques, on corrige la position de la bulle chaude. Par ailleurs, la bulle chaude étant parfaitement centrée, on risque moins l'invasion de la base du puits par de l'eau froide.

Aux températures envisagées $\left(180^{\circ}-190^{\circ} \mathrm{C}\right)$ les sources de chaleur sont actuellement les usines d'incinération d'ordures. Il s'agit d'un " marché relativement limité surtout si l'on ajoute les différentes contraintes, à savoir l'existence de la source, l'existence de la consommation et l'existence du terrain favorable " ( $M$. DESPOIS).

En fait, pour le CEA, les usines d'incinération constituent un marché d'attente, à la limite de la rentabilité ou même légèrement au-dessous, mais qui permet de maintenir les équipes en activité pour un développement futur qui est le transfert de l'été sur l'hiver de la chaleur excédentaire dans les centrales nucléaires (question de $M$. DESSUS - Président).

Dans le cas de Plaisir, l'étude économique a été faite en prenant comme prix de vente de la chaleur stockée celui du réseau existant, c'est-à-dire le prix du gaz moins $7 \%$ (question de $M$. IRIS ).

En ce qui concerne les problèmes chimiques, ils sont les mêmes à Plaisir qu’à Sarcelles (exposé de M. BoISDET) mais les installations sont plus petites; il y a plus de matière à retirer mais les débits à traiter sont plus faibles (question de M. DESSUS Président).
Il n'est pas nécessaire de faire un désiliçage avant le passage dans l'échangeur de récupération car, la silice restant facilement en sursaturation par rapport au quartz, il suffit que l'échangeur ait une température minimale au-dessus du seuil de formation de la silice amorphe. "Le problème le plus important n'est pas le problème de la silice elle-même, c'est le problème de la néoformation de kaolin au puits de réinjection, parce qu'il y a toujours, à ce moment là, des ions aluminium qui se promènent, provenant des autres argiles, et qui viennent s'associer avec la silice et former un colmatage très important au niveau du puits de réinjection. C'est une cause de colmatage contre laquelle on doit lutter " ( $M$. DESPOIS).

Tous les problèmes liés à l'injection dans un aquifère de type détritique ne sont pas résolus mais l'installation de traitements chimiques prévue est très élaborée, aucune hypothèse n'ayant été faite, quasiment tous les traitements sont possibles (question de M. MARTIN).

Dans les opérations ultérieures il devrait être possible de réduire cette installation afin de diminuer les coûts. Après le traitement chimique il y a filtrage d'abord par des filtres à sable, puis, si nécessaire, par des filtres à cartouche.

Au niveau des puits, une complétion très classique est utilisée (question de $M$. MARTIN). Différents types de gravillons et différentes granulométries sont à l'étude afin de résoudre les problèmes qui se posent principalement pour le puits central.

Quant au choix de l'aquifère, il avait été prévu d'utiliser l'Albien plutôt que le Néocomien dans une opération à caractère purement expérimental hors Ile de France, à Troyes. Il aurait été ainsi prouvé que l'on pouvait utiliser l'Albien sans conséquences néfastes. L'expérience n'a pu avoir lieu et, à Plaisir, la perméabilité de l'Albien était insuffisante donc c'est le Néocomien qui est utilié (question de $M$. LEJEUNE).

Par ailleurs, le fait de se trouver dans le même horizon aquifère que le stockage de gaz de Beynes a facilité, dans une certaine mesure, les démarches administratives car l'installation se trouve dans le périmètre éloigné de sécurité et ne peut donc pas condamner un aquifère qui l'est déjà (question de $M$. SAUTY). 